\title{
Controlling residual hydrogen gas in mass spectra during pulsed laser atom probe tomography
}

\author{
R. Prakash Kolli*i]
}

\begin{abstract}
Residual hydrogen $\left(\mathrm{H}_{2}\right)$ gas in the analysis chamber of an atom probe instrument limits the ability to measure $\mathrm{H}$ concentration in metals and alloys. Measuring $\mathrm{H}$ concentration would permit quantification of important physical phenomena, such as hydrogen embrittlement, corrosion, hydrogen trapping, and grain boundary segregation. Increased insight into the behavior of residual $\mathrm{H}_{2}$ gas on the specimen tip surface in atom probe instruments could help reduce these limitations. The influence of user-selected experimental parameters on the field adsorption and desorption of residual $\mathrm{H}_{2}$ gas on nominally pure copper (Cu) was studied during ultraviolet pulsed laser atom probe tomography. The results indicate that the total residual hydrogen concentration, $H_{\mathrm{TOT}}$, in the mass spectra exhibits a generally decreasing trend with increasing laser pulse energy and increasing laser pulse frequency. Second-order interaction effects are also important. The pulse energy has the greatest influence on the quantity $H_{\text {TOT, }}$, which is consistently less than 0.1 at.\% at a value of $80 \mathrm{pJ}$.
\end{abstract}

Keywords: Atom probe tomography (APT), Copper, Hydrogen, Laser pulsing

\section{Background}

Residual hydrogen $\left(\mathrm{H}_{2}\right)$ gas that is present in the analysis chamber of an atom probe instrument is typically detected in the mass spectrum of a specimen tip during analysis $[1,2]$. The gas directly adsorbs to the specimen tip surface between each voltage or laser pulse and can then be desorbed during a subsequent pulse. The gas molecules impact the specimen tip and rebound thereby losing a small amount of kinetic energy during each bounce and then bind to a protruding surface atom [3]. The presence of field adsorbed residual $\mathrm{H}_{2}$ gas can limit the ability to quantify $\mathrm{H}$ concentration in metals and alloys during solid-state phenomena, such as hydrogen embrittlement, corrosion, hydrogen trapping, and grain boundary segregation since it is difficult to distinguish between the two sources of $\mathrm{H}$. It also promotes the formation of metal hydride complex ions, $\mathrm{M}_{x} \mathrm{H}_{y}$, further complicating

\footnotetext{
*Correspondence: pkolli@umd.edu

Department of Materials Science and Engineering, University of Maryland,

2144 Chemical and Nuclear Engineering Bldg., \#090, College Park, MD 20742-2115, USA
}

quantitative analysis of elemental concentrations in the mass spectra [1,2]. Several recent studies have employed $\mathrm{H}$ charging where deuterium is introduced into the atom probe tomography (APT) specimen tip as a substitute for the $\mathrm{H}$ to be measured in an iron/vanadium $(\mathrm{Fe} / \mathrm{V})$ multilayer film [4], steels [5-7], silver ( $\mathrm{Ag})$ [7], zirconium ( $\mathrm{Zr}$ ) alloys [8], and silicon (Si) [9]. The quantity of residual $\mathrm{H}_{2}$ gas must, however, still be accounted for due to overlap in the mass spectra in order to estimate the $\mathrm{H}$ concentration. Hence, improved insight and understanding into the influence of experimental parameters on the behavior of residual $\mathrm{H}_{2}$ gas on the specimen tip surface in state-ofthe-art atom probe instruments could help reduce these limitations. Such an understanding may permit minimization of the quantity of field adsorbed residual $\mathrm{H}_{2}$ gas and thereby allow more accurate quantification of the $\mathrm{H}$ concentration in metals and alloys.

Several authors have investigated the relationship between $\mathrm{H}_{2}$ gas adsorption and desorption and electric field during pulsed voltage one-dimensional (1D) atom probe field ion microscope (APFIM) analysis of materials including a nickel-rich (Ni-rich) alloy [10], tungsten 
(W) [11, 12], aluminum (Al) [13], and copper $(\mathrm{Cu})$ [13]. Iron $(\mathrm{Fe}), \mathrm{Ni}, \mathrm{Cu}$, palladium (Pd), molybdenum (Mo), $\mathrm{W}$, and platinum $(\mathrm{Pt})$ were also investigated as part of two broader studies [14, 15]. Some of the aforementioned studies illustrated that the $\mathrm{H}$ signal decreases with increasing field strength [11], or that a critical DC field strength exists depending on the metal or alloy, above which the $\mathrm{H}$ signal decreases with increasing field strength $[10,12]$.

Some studies on the effect of experimental parameters on $\mathrm{H}_{2}$ gas field adsorption and desorption using laserpulsed mode in an atom probe instrument have been performed [16-18]. More specifically, field adsorption and desorption of $\mathrm{H}_{2}$ gas on molybdenum (Mo) during 1D-pulsed laser atom probe (PLAP) experiments equipped with a nitrogen laser (337 $\mathrm{nm}$ wavelength) was previously investigated [16]. This study evaluated the $\mathrm{H}$ signal intensity at substrate (base) temperatures between 100 and $150 \mathrm{~K}$ and at slow pulse rates between 1 and $60 \mathrm{~Hz}$. An investigation on the field adsorption of $\mathrm{H}_{2}$ gas on W during 1D PLAP experiments was also performed [17] and evaluated the $\mathrm{H}$ signal intensity at temperatures between $\sim 80$ and $250 \mathrm{~K}$. The pulse rates accessible by the instruments used in these studies are significantly slower than that attainable in today's state-of-the-art instruments, and the base temperatures studied were greater than used today. More recently, the influence of laser pulse energy on field adsorption of residual $\mathrm{H}_{2}$ gas was evaluated on a nickel-chromium $(\mathrm{Ni}-\mathrm{Cr})$ alloy when using a Local-Electrode Atom-Probe $\left(\right.$ LEAP $\left.^{\circledR}\right)$ instrument equipped with a green laser (532 nm wavelength) [18].

The high mass resolving power, $m / \Delta m$, and high elemental sensitivity in state-of-the-art atom probe instruments $[19,20]$ make performing APT measurements of $\mathrm{H}$ concentration in metals and alloys feasible. However, no studies have been performed to study the influence of user-selected experimental parameters in modern atom probe instruments equipped with a UV $(355 \mathrm{~nm}$ wavelength) laser on the quantity of residual $\mathrm{H}_{2}$ gas detected in mass spectra. Furthermore, limited earlier studies using laser-pulsed mode were reported in the extant literature, and few have evaluated low temperatures $<100 \mathrm{~K}$, or fast pulse rates $\geq 100 \mathrm{kHz}$, or the combined effect of user-selected experimental parameters. Typically studies were performed to evaluate a single parameter. Additionally, $\mathrm{Cu}$ is an important electrical and structural material that experiences the aforementioned solid-state phenomena and studies on controlling residual $\mathrm{H}_{2}$ gas field adsorption and desorption on $\mathrm{Cu}$ have not been reported in the literature. Improved insight and understanding into the influence of experimental parameters in state-of-the-art atom probe instruments on the total residual hydrogen concentration, $H_{\mathrm{TOT}}$, that is detected may permit minimization of the gas in mass spectra. This study evaluates the synergistic laser pulse energy, laser pulse frequency, and specimen tip base temperature effects on the quantity $H_{\text {TOT }}$ that is detected during UVpulsed laser APT of nominally pure $\mathrm{Cu}$. The results are discussed in context of a constant evaporation rate theoretical model in order to evaluate the relative influence of the three aforesaid parameters. A statistical analysis was also performed to ascertain the significance of each parameter and their interactions on the quantity $H_{\text {TOT }}$. Other $\mathrm{H}$ sources that can influence the quantity $H_{\mathrm{TOT}}$ are discussed including the possibility of impingement and dissociation of $\mathrm{H}_{2} \mathrm{O}$ on the specimen tip and surface diffusion of residual $\mathrm{H}_{2}$ gas molecules. The short-range binding energy of a $\mathrm{H}_{2}$ molecule on a $\mathrm{Cu}$ metal surface atom is estimated based on the model presented in Refs. $[21,22]$, and the mechanisms to form the $\mathrm{H}^{+}$and $\mathrm{H}_{3}^{+}$ions detected in the mass spectra are discussed in context of the binding energy. We discuss how the residual $\mathrm{H}_{2}$ gas in the mass spectra can be minimized and the broader applicability of the results to other materials.

\section{Experimental methods}

A $0.5 \mathrm{~mm}$ diameter 99.999 wt.\% polycrystalline $\mathrm{Cu}$ wire taken from Standard Reference Material (SRM) 482 of the National Institute of Standards and Technology (NIST) was used as the source material. Specimens with a needle-shaped geometry necessary for APT analysis were fabricated using a FEI Nova 600 dual-beam scanning electron microscope/focused ion beam (SEM/ FIB) instrument following standard lift-out and annular milling procedures [23-25]. A platinum $(\mathrm{Pt})$ protective layer was deposited over a region of interest (ROI) using established procedures. Annular milling was performed employing a $30 \mathrm{kV}$ gallium ion $\left(\mathrm{Ga}^{+}\right)$beam and sequentially decreasing probe current following standard procedures after transfer of the ROI to silicon $(\mathrm{Si})$ microtip posts with an Omniprobe Autoprobe 200 Micromanipulator. A low $\mathrm{kV}$ ion beam of $5 \mathrm{kV}$ was allowed to raster over the specimen tip to remove material that had been damaged by the $30 \mathrm{kV} \mathrm{Ga}^{+}$ion beam annular milling operation [26].

Pulsed laser APT was performed employing a CAMECA LEAP ${ }^{\circledR} 4000 \mathrm{X}$ instrument in the Si configuration with a $355 \mathrm{~nm}$ UV-pulsed laser under ultra-high vacuum (UHV) conditions of $1.2 \times 10^{-8} \mathrm{~Pa}\left(9 \times 10^{-11}\right.$ Torr). A full factorial $3 \times 3 \times 3$ experimental matrix was used where the laser pulse energy was set at 40,60 , or $80 \mathrm{pJ}$; the laser pulse frequency at 100,250 , or $500 \mathrm{kHz}$; and the specimen tip base temperature at 20,50 , or $80 \mathrm{~K}$ leading to 27 individual experiments on different specimen tips. The nominal detection rate was maintained at a constant 0.01 ions per pulse or $1 \%$. Datasets of greater than two 
million ions were collected for each experiment. Mass spectra analysis was performed using the CAMECA Integrated Visualization and Analysis Software $\left(\right.$ IVAS $\left.^{\circledR}\right)$, version 3.6.6. Statistical analysis of the data by analysis of variance (ANOVA) using the $F$ test with a significance of $\alpha=0.05$ was performed employing the $\mathrm{JMP}^{\circledR}$ software, version 12

\section{Results}

\section{Mass spectrum}

Sections of a background-noise-corrected example mass spectrum are illustrated in Fig. 1a-d. Hydrogen is present as atomic hydrogen, $\mathrm{H}^{+}$, at a mass-to-charge state $(m / n)$ ratio of $1 \mathrm{Da}$, and also as molecular hydrogen, $\mathrm{H}_{2}^{+}$ and $\mathrm{H}_{3}^{+}$, at $m / n$ ratios of 2 and $3 \mathrm{Da}$. The peak at a $m / n$ ratio of $1 \mathrm{Da}$ may also contain $\mathrm{H}_{2}^{2+}$ ions. The $\mathrm{H}_{3}^{+}$peak is small relative to the $\mathrm{H}^{+}$and $\mathrm{H}_{2}^{+}$peaks in this example mass spectrum but can have a greater number of counts depending on the user-selected experimental parameters. Hydrogen is also present as a complex ion at a $m / n$ ratio of $67 \mathrm{Da}$, which we have ascribed to $\mathrm{CuH}_{2}^{1+}$. This complex ion should also be present at a $m / n$ ratio of $65 \mathrm{Da}$ but it is convoluted with ${ }^{65} \mathrm{Cu}^{1+}$. Oxygen $(\mathrm{O})$ is present at a $m / n$ ratio of $16 \mathrm{Da}$ and as molecular oxygen, $\mathrm{O}_{2}^{1+}$, at a $m / n$ ratio of $32 \mathrm{Da}$. Oxygen is also present as part of complex ions at $m / n$ ratios of 39.5 and $79 \mathrm{Da}$, which we
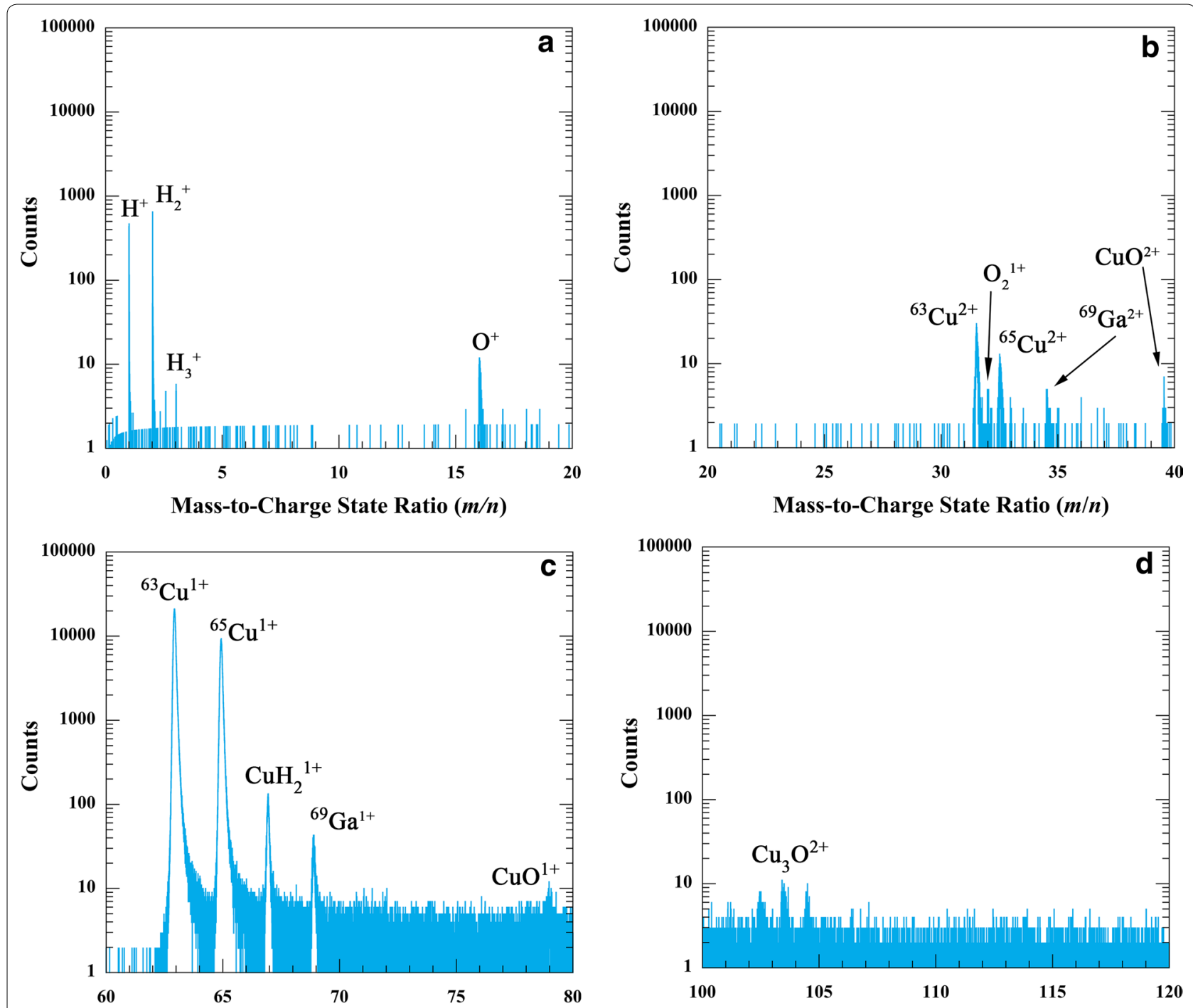

Mass-to-Charge State Ratio ( $m / n)$

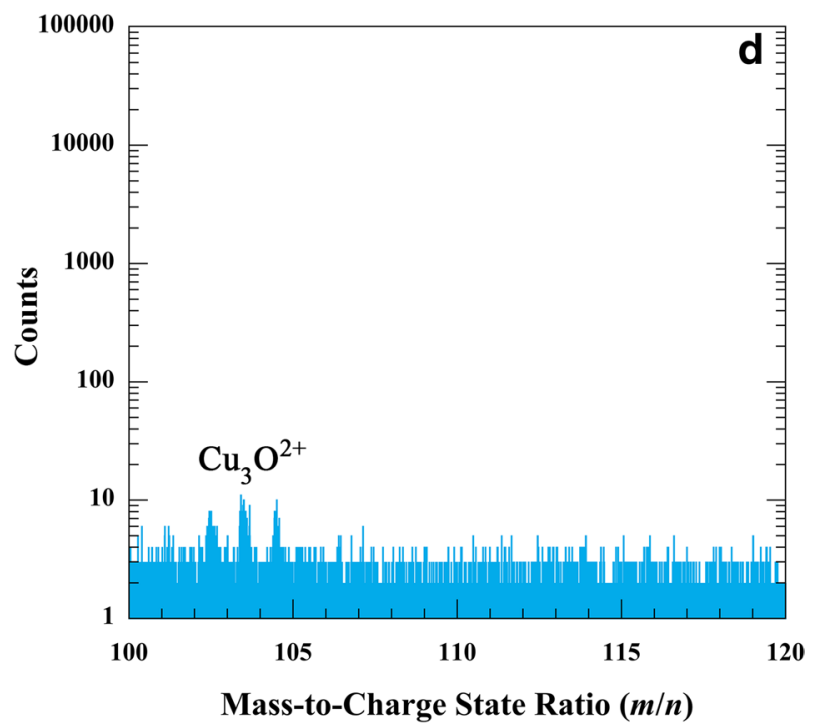

Fig. 1 Sections of an example mass spectrum illustrate the presence of $\mathbf{a ~} \mathrm{H}^{+}, \mathrm{H}_{2}^{+}, \mathrm{H}_{3}^{+}$, and $\mathrm{O}^{+}$peaks; $\mathbf{b} \mathrm{Cu}^{2+}, \mathrm{O}_{2}^{1+}, \mathrm{Ga}^{2+}$, and $\mathrm{CuO}^{2+}$ peaks; $\mathbf{c} \mathrm{Cu}^{+}$,

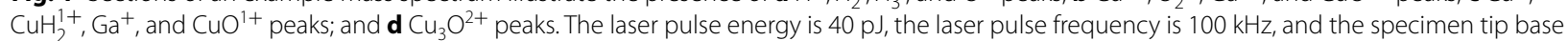
temperature is $20 \mathrm{~K}$ 
have ascribed to $\mathrm{CuO}^{2+}$ and $\mathrm{CuO}^{1+}$, respectively, and at $m / n$ ratios of $102.5,103.5$, and $104.5 \mathrm{Da}$, which we have ascribed to $\mathrm{Cu}_{3} \mathrm{O}^{2+}$. The complex ions were identified through ion correlation histograms, which are twodimensional (2D) histograms of the ion pairs in multi-hit ion detection events $[27,28]$, and illustrated for $\mathrm{Cu}$ in Refs. [29, 30]. Additionally, the expected peaks for $\mathrm{Cu}$ at $m / n$ ratios of 63 and $65 \mathrm{Da}$ for the $1+$ charged state and at $m / n$ ratios of 31.5 and $32.5 \mathrm{Da}$ for the $2+$ charged states are present. Gallium is present in small quantities at $m / n$ ratios of 69 and $34.5 \mathrm{Da}$ for the $1+$ and $2+$ charged states, respectively.

\section{Hydrogen}

The quantity $H_{\text {TOT }}$ is the sum of the atomic, molecular, and complex ion hydrogen and thus includes $\mathrm{H}^{+}, \mathrm{H}_{2}^{+}, \mathrm{H}_{3}^{+}$, and $\mathrm{CuH}_{2}^{1+}$. All the ions at a $m / n$ ratio of $1 \mathrm{Da}$ are assigned to $\mathrm{H}^{+}$since the detected quantity of $\mathrm{H}_{2}^{2+}$ is expected to be minimal, as discussed below. Since hydrogen in the $\mathrm{CuH}_{2}^{1+}$ complex ion at a $m / n$ ratio of $65 \mathrm{Da}$ is convoluted with ${ }^{65} \mathrm{Cu}^{1+}$, the quantity of $\mathrm{H}$ at this peak contributing to the quantity $H_{\mathrm{TOT}}$ is determined based upon the isotopic abundance ratio of $\mathrm{Cu}$, i.e., $\mathrm{CuH}_{2}^{1+}$ is detected in the same abundance ratio as $\mathrm{Cu}$. Measureable quantities of $\mathrm{H}$ are still present in the mass spectra despite performing the experiments at UHV typical of state-of-the-art atom probe analysis. The quantity $H_{\mathrm{TOT}}$ in the mass spectra is illustrated as a function of laser pulse energy at specimen tip base temperatures of $20 \mathrm{~K}$ (red triangles), $50 \mathrm{~K}$ (green squares), and $80 \mathrm{~K}$ (blue diamonds); and laser pulse frequencies of $100 \mathrm{kHz}$ (solid symbols), $250 \mathrm{kHz}$ (half-filled symbols), and $500 \mathrm{kHz}$ (open symbols), Fig. 2. The quantity $H_{\mathrm{TOT}}$ demonstrates a decreasing trend between 40 and $80 \mathrm{pJ}$ pulse energies at these base temperatures and pulse frequencies of 100 and $250 \mathrm{kHz}$. The relationship is more complex at a pulse frequency of $500 \mathrm{kHz}$. At a base temperature of $20 \mathrm{~K}$, a decreasing trend is observed in the quantity $H_{\mathrm{TOT}}$ between 40 and $80 \mathrm{pJ}$. At a base temperature of $50 \mathrm{~K}$, a decreasing trend in the quantity $H_{\text {TOT }}$ is observed between 40 and $60 \mathrm{pJ}$, and it is then approximately constant to $80 \mathrm{pJ}$. More variability is observed when the base temperature is $80 \mathrm{~K}$. Further, the quantity $H_{\text {TOT }}$ decreases with increasing pulse frequency at pulse energies of 40 and $60 \mathrm{pJ}$ and at a base temperature of 20 and $50 \mathrm{~K}$ but some variability is again observed at a temperature of $80 \mathrm{~K}$. Less difference is exhibited in the quantity $H_{\mathrm{TOT}}$ between frequencies at a pulse energy of $80 \mathrm{pJ}$.

The quantity $H_{\mathrm{TOT}}$ is also illustrated as a function of laser pulse frequency at specimen tip base temperatures of $20 \mathrm{~K}$ (red triangles), $50 \mathrm{~K}$ (green squares), and $80 \mathrm{~K}$ (blue diamonds) and at laser pulse energies of $40 \mathrm{pJ}$ (solid symbols), $60 \mathrm{pJ}$ (half-filled symbols), and 80 pJ (open symbols), Fig. 3. The quantity $H_{\mathrm{TOT}}$ demonstrates a decreasing trend with

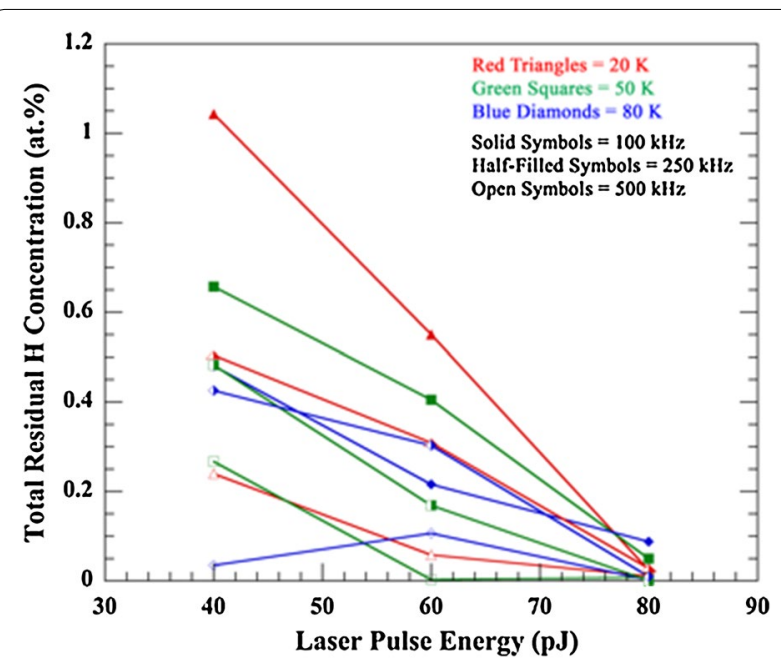

Fig. 2 The total residual $\mathrm{H}$ concentration, $H_{\text {TOT, }}$ (at.\%) plotted as a function of laser pulse energy between 40 and $80 \mathrm{pJ}$. The specimen tip base temperatures are $20 \mathrm{~K}$ (red triangles), $50 \mathrm{~K}$ (green squares), or $80 \mathrm{~K}$ (blue diamonds), and the laser pulse frequencies are $100 \mathrm{kHz}$ (solid symbo/s), $250 \mathrm{kHz}$ (half-filled symbo/s), and $500 \mathrm{kHz}$ (open symbo/s). The $\pm 2 \sigma$ error bars, which are based on counting statistics, are not visible since they are smaller than the size of the data point markers

increasing pulse frequency at a pulse energy of 40 and $60 \mathrm{pJ}$ and at these base temperatures, although some variability is observed at $80 \mathrm{~K}$. The pulse frequency has a much smaller effect on the quantity $H_{\text {TOT }}$ at a pulse energy of $80 \mathrm{pJ}$, which is approximately constant between 250 and $500 \mathrm{kHz}$ at all three base temperatures. A slightly decreasing trend is, however, observed with increasing frequency between 100 and $250 \mathrm{kHz}$ at 50 and $80 \mathrm{~K}$, whereas it is approximately constant at $20 \mathrm{~K}$. Additionally, the specimen tip base temperature has less effect on the quantity $H_{\text {TOT }}$ at an $80 \mathrm{pJ}$ pulse energy when compared to 40 and $60 \mathrm{pJ}$. Increasing the pulse energy at a pulse frequency of 100 or $250 \mathrm{kHz}$ and a given base temperature reduces the quantity $H_{\mathrm{TOT}}$. The relationship is more complex at a frequency of $500 \mathrm{kHz}$.

In order to determine the significance of each experimental parameter, ANOVA was performed on the data with the quantity $H_{\text {TOT }}$ as the response variable. The observed nonlinearities and variability at certain experimental parameter combinations in Figs. 2 and 3 suggest that complex interactions between the parameters may also influence the quantity $H_{\mathrm{TOT}}$, and these were also evaluated. The main experimental parameters of laser pulse energy $(F=137.1, P<0.001)$, laser pulse frequency $(F=68.9, P<0.001)$, and base temperature $(F=10.9$, $P<0.004)$ were significant. Additionally, ANOVA illustrated as significant the second-order interactions of pulse energy and pulse frequency $(F=30.2, P<0.001)$, pulse energy and base temperature $(F=10.4, P<0.001)$, and pulse frequency and base temperature $(F=4.7$, 


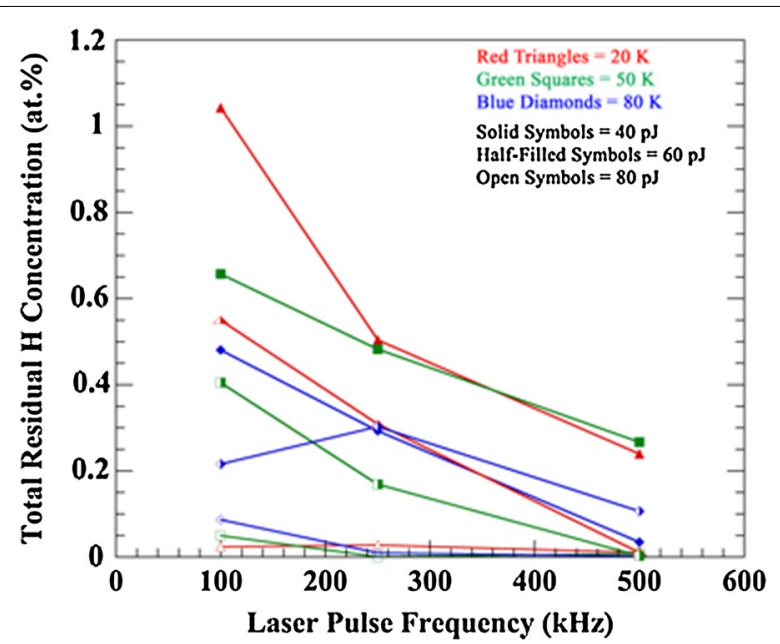

Fig. 3 The total residual $\mathrm{H}$ concentration, $H_{\text {TOT, }}$ (at.\%) plotted as a function of laser pulse frequency between 100 and $500 \mathrm{kHz}$. The specimen tip base temperatures are $20 \mathrm{~K}$ (red triangles), $50 \mathrm{~K}$ (green squares), or $80 \mathrm{~K}$ (blue diamonds), and the laser pulse energies are $40 \mathrm{pJ}$ (solid symbo/s), $60 \mathrm{pJ}$ (half-filled symbo/s), and $80 \mathrm{pJ}$ (open symbo/s). The $\pm 2 \sigma$ error bars, which are based on counting statistics, are not visible since they are smaller than the size of the data point markers

$P<0.042)$. Contour plots of the experimental parameters illustrate their complex interaction at constant values of $H_{\mathrm{TOT}}$, Fig. $4 \mathrm{a}-\mathrm{c}$. The plots also illustrate the dependence of the quantity $H_{\mathrm{TOT}}$ on the experimental parameters.

\section{Discussion}

Influence of the experimental parameters on total residual $\mathrm{H}$ concentration

The results indicate that laser pulse energy has greater influence on the quantity $H_{\mathrm{TOT}}$ when compared to laser pulse frequency and specimen tip base temperature. Increasing the laser pulse energy from 40 to $80 \mathrm{pJ}$ significantly lowers the quantity $H_{\mathrm{TOT}}$. At a $80 \mathrm{pJ}$ pulse energy, the quantity $H_{\text {TOT }}$ is consistently less than 0.1 at.\% regardless of the choice of pulse frequency or base temperature within the studied range of experimental parameters. This value is lower than the $\sim 0.2$ at.\% attained for the total $\mathrm{H}$ concentration when employing a green laser at $\sim 600 \mathrm{pJ}$ in Ref. [18]. Increasing the laser pulse frequency from 100 to $500 \mathrm{kHz}$ also lowers the quantity $H_{\text {TOT }}$. The approximately linear relationship between the quantity $H_{\mathrm{TOT}}$ and increasing laser pulse frequency at a $40 \mathrm{pJ}$ pulse energy and at a $50 \mathrm{~K}$ base temperature and also at $60 \mathrm{pJ}$ and 20 or $50 \mathrm{~K}$ are consistent with the observations between 1 and $60 \mathrm{~Hz}$ in Ref. [16], and 50 and $200 \mathrm{kHz}$ in Ref. [18] thereby indicating that the reported linear proportionality extends to higher pulse frequencies. However, a similar linear relationship is not observed when the pulse energy is $80 \mathrm{pJ}$, which may be due to its greater influence and second-order interaction effects. Further, the predominant effect of pulse frequency during field adsorption is to affect the time available for adsorption of residual $\mathrm{H}_{2}$ gas molecules between each pulse, i.e., a faster pulse frequency will reduce the time available. Hence, a faster pulse frequency will lead to a lower amount of surface coverage of an adsorbing gas than at a slower pulse frequency thereby leading to a lower value for the quantity $H_{\mathrm{TOT}}$. The base temperature has the least influence on the quantity $H_{\mathrm{TOT}}$, especially when the pulse energy is $80 \mathrm{pJ}$, and the pulse frequency is $500 \mathrm{kHz}$.

The approximately constant relationship or only small variation of the quantity $H_{\mathrm{TOT}}$ as a function of pulse frequency at a $80 \mathrm{pJ}$ pulse energy, and a given specimen tip base temperature suggests a change in physical behavior that is possibly related to the greater influence of pulse
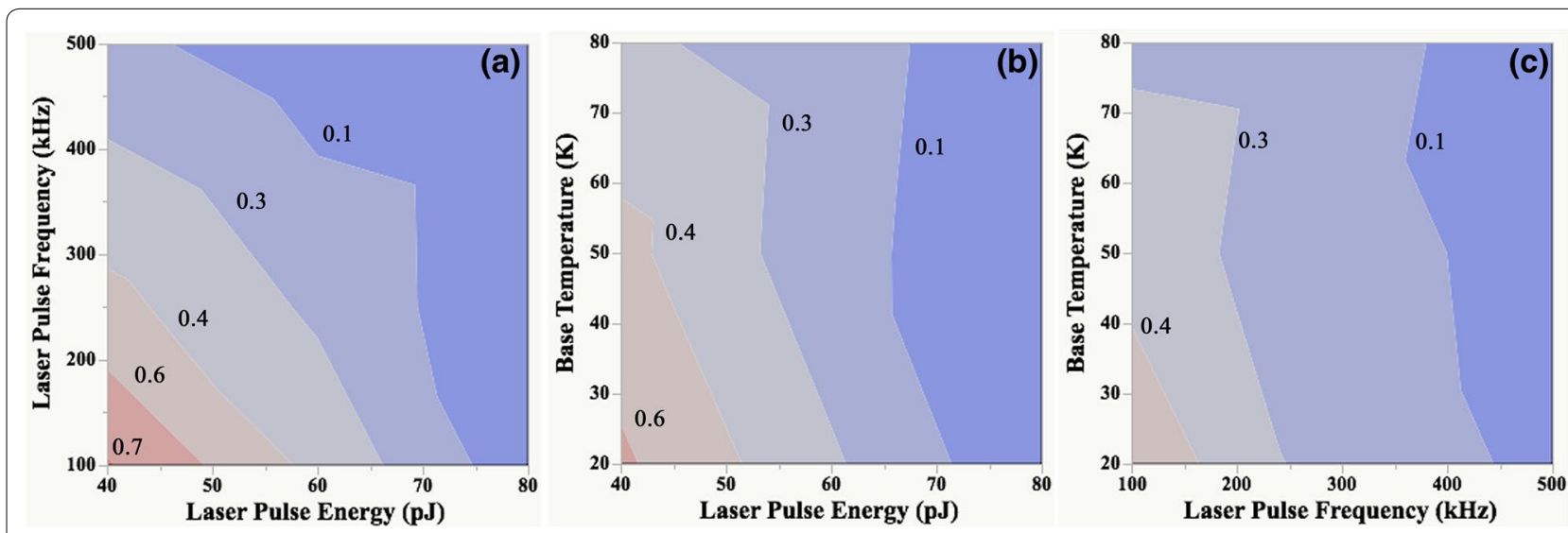

Fig. 4 Contour plots illustrating the interaction of the experimental parameters $\mathbf{a}$ laser pulse frequency and laser pulse energy, $\mathbf{b}$ base temperture and laser pulse energy, and $\mathbf{c}$ base temperature and laser pulse frequency at a constant value of total residual $\mathrm{H}$ concentration, $H_{\text {TOT, }}$ (at.\%). Lower values of the quantity $H_{\text {Tот }}$ are represented by cool coloration (purple and blue) and higher values are represented by warm coloration (pink and red) 
energy. The measured dependence of the quantity $H_{\text {TOT }}$ on the experimental parameters suggests a relationship between these parameters and the specimen tip apex temperature at a constant evaporation rate. The evaporation rate at time $t, \Phi(t)$, due to the electric field strength derived from the applied DC voltage, $F_{0}$, specimen tip base temperature, $T_{0}$, and temperature rise of the specimen tip apex due to laser pulsing, $T_{\text {rise }}$, can be expressed as [31]

$$
\Phi(t) \approx v N \tau_{\mathrm{t}} \exp \left(\frac{-Q_{n}\left(F_{0}\right)}{k_{\mathrm{B}}\left(T_{0}+T_{\text {rise }}\right)}\right),
$$

where $v$ is the vibrational energy of the $N$ kink site atoms that can be field evaporated, $\tau_{\mathrm{t}}$ is the temporal width of the temperature profile, $Q_{n}$ is the activation energy that is a function of $F_{0}$, and $k_{\mathrm{B}}$ is Boltzmann's constant. At a constant evaporation rate, the field strength is greater in value at a lower specimen tip apex temperature, $T_{0}+T_{\text {rise }}$, that are characteristic of low-pulse energies. Conversely, the field strength is lower in value at a higher tip apex temperature that are characteristic of high-pulse energies since the quantity $T_{\text {rise }}$ increases with increasing laser pulse energy [32-35]. Thus, since the quantity $H_{\mathrm{TOT}}$ is related to the quantity $F_{0}[16,18]$, it is also related to the quantity $T_{0}+T_{\text {rise }}$ through Eq. (1). The quantity $T_{\text {rise }}$ has been estimated as between 160 and $300 \mathrm{~K}$ during normal APT operating conditions [34,36], which is greater than the quantity $T_{0}$ and hence suggests that the laser pulse energy has a greater influence on the quantity $H_{\text {TOT }}$.

The second-order interaction between laser pulse energy and laser pulse frequency is also important in reducing the quantity $H_{\mathrm{TOT}}$, which is possibly due to both parameters influence on the specimen tip apex temperature combined with the latter's effect on residual $\mathrm{H}_{2}$ gas surface coverage. We discuss above the pulse energy effect on tip apex temperature and pulse frequency may also affect it since it directly correlates to cooling time between pulses $[29,30]$. The second-order interactions between base temperature and laser pulse energy and also laser pulse frequency are less important and have less effect on reducing the quantity $H_{\text {TOT }}$.

The combination of a $80 \mathrm{pJ}$ laser pulse energy, $500 \mathrm{kHz}$ laser pulse frequency, and a base temperature between 20 and $80 \mathrm{~K}$ leads to a value for the quantity $H_{\text {TOT }}<0.1$ at.\%. The combination of a $80 \mathrm{pJ}$ laser pulse energy, $250 \mathrm{kHz}$ laser pulse frequency, and $20 \mathrm{~K}$ specimen tip base temperature leads to a value for the quantity $H_{\mathrm{TOT}}<0.1$ at.\%, and also improved mass resolving power and smaller tails after major single-charged state peaks in $\mathrm{Cu}$ based on prior results in Refs. [29, 30].

\section{Residual $\mathrm{H}_{\mathbf{2}}$ gas supply mechanism}

Three sources of residual $\mathrm{H}_{2}$ gas that adsorb to the APT specimen tip may occur: (1) direct adsorption of $\mathrm{H}_{2}$ gas,
(2) impingement and dissociation of $\mathrm{H}_{2} \mathrm{O}$ molecules, and possibly (3) surface diffusion of $\mathrm{H}_{2}$ gas along the shank of the tip due to a thermal or field gradient as suggested in Refs. [10, 18]. Direct adsorption of residual $\mathrm{H}_{2}$ gas from the analysis chamber is addressed above. In addition, the experimental observations suggest that small quantities of $\mathrm{H}_{2} \mathrm{O}$ impingement and dissociation may be exhibited in some specimen tips. Although a $\mathrm{H}_{2} \mathrm{O}^{+}$peak is not observed at a $m / n$ ratio of 18 in Fig. 1a, this peak is present in the mass spectrum of some specimen tips. Furthermore, $\mathrm{a} \mathrm{OH}^{+}$peak is observed in the mass spectrum at a $m / n$ ratio of 17 indicating that dissociation of $\mathrm{H}_{2} \mathrm{O}$ is occurring. In addition to the complex ions consisting of $\mathrm{Cu}, \mathrm{H}, \mathrm{O}$, and $\mathrm{O}_{2}$ combinations as described above, complex ion peaks at $m / n$ ratios of 80 and 82 that correspond to $\mathrm{CuOH}^{+}$are also observed in the mass spectrum when $\mathrm{H}_{2} \mathrm{O}$ impingement and dissociation occurs. The contribution of this source to the quantity $H_{\mathrm{TOT}}$ is, however, small when compared to that of direct adsorption of $\mathrm{H}_{2}$ gas.

The experimental observations preclude the possibility of significant residual $\mathrm{H}_{2}$ gas surface diffusion along the shank of the tip from its base to its apex due to a thermal gradient. To a first approximation, surface diffusion obeys Arrhenius behavior where the diffusivity (or the diffusion coefficient) increases with increasing temperature as

$$
D=D_{0} \exp \left(\frac{-Q_{\mathrm{s}}}{R T}\right),
$$

where $D_{0}$ is the pre-exponential constant; $Q_{\mathrm{S}}$ is the activation energy for surface diffusion; $R$ is the gas constant; and $T$ is the absolute temperature. If surface diffusion due to thermal gradient was a significant mechanism to supply $\mathrm{H}_{2}$ gas to the tip apex, the quantity $H_{\text {TOT }}$ should increase with increasing pulse energy at a given specimen tip base temperature and pulse frequency, which is not observed in the results. Alternatively, the increase in temperature at the specimen tip apex due to increased laser pulse energy concomitantly reduces the field at this location and thus the field gradient between the tip apex and its base. This in turn may cause a reduction in the supply of residual $\mathrm{H}_{2}$ gas by surface diffusion to the tip apex that corresponds to a reduction in the quantity $H_{\text {TOT }}$. The field strength at the specimen tip base and shank is, however, less than at the tip apex thereby suggesting that limited residual $\mathrm{H}_{2}$ gas adsorbs at these locations. Residual $\mathrm{H}_{2}$ gas may also be adsorbed at the specimen tip base and shank due to trapping at cryogenic temperatures, i.e., a "cold finger" mechanism. But significant quantities of $\mathrm{H}_{2}$ are not trapped until temperatures are less than $\sim 10 \mathrm{~K}$, which is lower than the specimen tip base temperatures in this study. Thus, surface diffusion along the shank of the tip from its base to its apex due to field gradient most 
likely has limited contribution to the residual $\mathrm{H}_{2}$ gas detected in a mass spectrum and the quantity $H_{\text {TOT }}$ when compared to that of direct adsorption of $\mathrm{H}_{2}$ gas.

\section{Short-range binding energy}

The short-range binding energy is estimated employing the model developed by Tsong and Müller [21, 22]. These authors suggested that field adsorption occurs due to short-range field-induced dipole-dipole interactions between the gas molecules and the apex of protruding metal surface atoms, where short-range is defined as an atomic radius or less $[21,22]$. This mechanism means that the protruding metal surface atoms, whether kink site or step site atoms, and the adsorbing species are polarizable due to the applied DC electric field. In this model, the binding energy, $\mathrm{H}_{\mathrm{A}}$, is

$$
\frac{1}{2} \alpha_{\mathrm{A}}\left(f_{\mathrm{A}}-1\right) F_{0}^{2},
$$

where $\alpha_{\mathrm{A}}$ is the polarizability of the gas atom or molecule; $f_{\mathrm{A}}$ is the enhancement factor due to field-induced dipole-dipole interactions; and $F_{0}$ is the applied electric field strength in $\mathrm{V} / \AA$. The quantity $f_{\mathrm{A}}$ is given by

$$
f_{\mathrm{A}}=\frac{\left(1+2 \alpha_{\mathrm{M}} / d^{3}\right)^{2}}{\left(1-4 \alpha_{\mathrm{M}} \alpha_{\mathrm{A}} / d^{6}\right)^{2}},
$$

where $\alpha_{\mathrm{M}}$ is the polarizability of the metal surface atom, and $d$ is the equilibrium distance of the interacting surface atom and the adsorbing species. The polarizability is related to the Gaussian or static electric dipole polarizability by the factor $4 \pi \varepsilon_{0}$, where $\varepsilon_{0}$ is the vacuum permittivity in free space. This model is used here since it reportedly has good correlation to experimentally measured values of short-range binding energies [37, 38], despite its differences in underlying theory with the image dipole model developed by Forbes [37-39], and the combination of short-range field-induced polarization bonding and field-induced covalent bonding model developed by Krezuer [38].

The short-range binding energy of a $\mathrm{H}_{2}$ gas molecule above a $\mathrm{Cu}$ metal surface atom is estimated using Eqs. (3) and (4). These equations assume that mutual polarization between adjacent dipoles in the gas and metal substrate and secondary interactions between the adsorbing species and surface atoms are negligible. These factors lead to only a small improvement of the short-range binding energy calculations in this model [37]. Additionally, the above equations assume that the kinetic energy gained by the ions as they move from a distant point to their binding point is negligible. This factor also leads to only a small enhancement of the short-range binding energy calculations in this model [37]. Thus, the first-order approximation that employs Eqs. (3) and (4) is a reasonable one to estimate the short-range binding energy of a $\mathrm{H}_{2}$ gas molecule on a $\mathrm{Cu}$ metal surface atom. The Gaussian polarizability of $\mathrm{Cu}, \alpha_{\mathrm{Cu}}$, is $5.0 \AA^{3}$ [40], the atomic radius of $\mathrm{Cu}, r_{\mathrm{Cu}}$, is $0.135 \mathrm{~nm}$ [41], and the Gaussian polarizability of $\mathrm{H}_{2}, \alpha_{\mathrm{H}_{2}}$, is $0.79 \AA^{3}$ [16]. The calculated shortrange binding energy for a $\mathrm{H}_{2}$ molecule as a function of the field strength after converting the Gaussian polarizability to standard SI units is illustrated in Fig. 5, where $d=r_{\mathrm{Cu}}+r_{\mathrm{H}}=0.255 \mathrm{~nm}$. An earlier estimate of the field strength that is required for field-induced adsorption of a $\mathrm{H}_{2}$ molecule on a $\mathrm{Cu}$ surface atom during pulsed voltage 1D APFIM was $2.05 \mathrm{~V} / \AA$ [13]. This field strength value suggests that the short-range binding energy is approximately $0.21 \mathrm{eV}$ using Fig. 5 .

\section{$\mathrm{H}^{+}$ion dissociation and $\mathrm{H}_{3}^{+}$ions}

The $0.21 \mathrm{eV}$ value for the quantity $\mathrm{H}_{\mathrm{A}}$ of a $\mathrm{H}_{2}$ molecule on a $\mathrm{Cu}$ surface atom is approximately an order of magnitude less than the reported binding energy of between 2.0 and $3.3 \mathrm{eV}$ for a $\mathrm{H}$ atom on a $\mathrm{Cu}$ surface atom $[42,43]$. The high values for the atomic $\mathrm{H}$ binding energy suggests that it would not be easily desorbed from the specimen tip during the thermal pulse. Since the quantity $\mathrm{H}_{\mathrm{A}}$ of molecular $\mathrm{H}_{2}$ is significantly less than the binding energy of atomic $\mathrm{H}$, it is likely that the $\mathrm{H}^{+}$ions detected in the mass spectrum at a $m / n$ ratio of $1 \mathrm{Da}$ occurred due to desorption of $\mathrm{H}_{2}^{+}$ions followed by ionization and dissociation of a subset of these ions in the high field surrounding the tip apex, i.e., $\mathrm{H}_{2}^{+} \rightarrow \mathrm{H}_{2}^{2+} \rightarrow \mathrm{H}^{+}+\mathrm{H}^{+}$.

The presence of $\mathrm{H}_{3}^{+}$ions in the example mass spectrum of Fig. 1a requires further discussion. Although neutral $\mathrm{H}_{3}$ is unstable in free space, it may be stable in the presence of an electric field. The $\mathrm{H}_{3}^{+}$ion reportedly has a

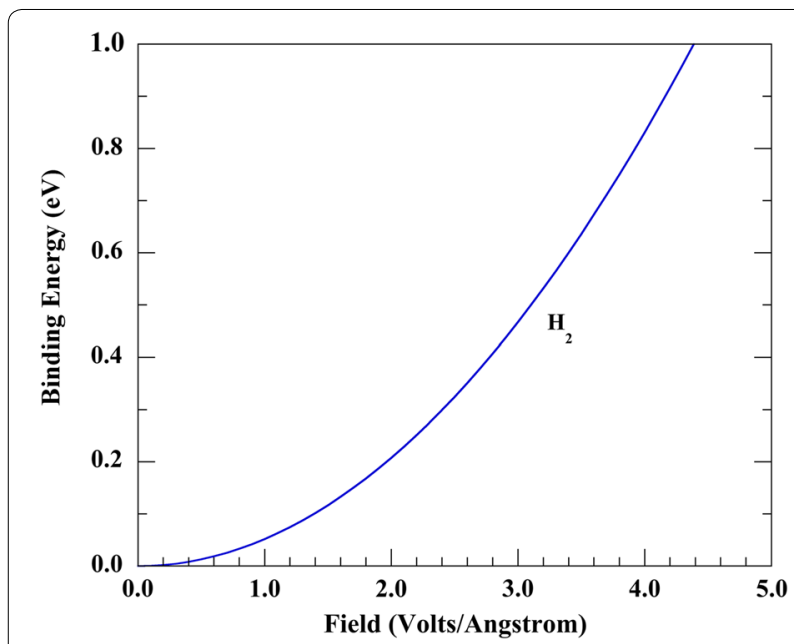

Fig. 5 The estimated short-range binding energy for field adsorbed residual $\mathrm{H}_{2}$ gas (blue line) on a copper (Cu) metal surface atom as a function of the field strength 
stable configuration in the electric field and after desorption $[44,45]$. It also does not easily field dissociate until a field strength of approximately $3.0 \mathrm{~V} / \AA[44,45]$, and thus, it can be detected in the mass spectra. Furthermore, the quantity of measured $\mathrm{H}_{3}^{+}$ions depends on the metal surface atom's crystallographic structure, field strength, laser pulse energy, and the specimen tip apex temperature $[1,46,47]$. For example, elements with a face-centered cubic (f.c.c.) crystal structure, such as $\mathrm{Cu}$, exhibit lower quantities of $\mathrm{H}_{3}^{+}$ions in a mass spectrum when compared to elements with hexagonal close-packed (h.c.p.) and body-centered cubic (b.c.c.) crystal structures. The experimental observations in this study are consistent with those in Refs. $[1,46,47]$ that indicate the quantity of $\mathrm{H}_{3}^{+}$ions measured in the mass spectra is dependent on the experimental parameters. For example, approximately $223 \mathrm{H}_{3}^{+}$ions are measured when the laser pulse energy is $40 \mathrm{pJ}$, and ca. $0 \mathrm{H}_{3}^{+}$ions when the pulse energy is $80 \mathrm{pJ}$, at a laser pulse frequency of $250 \mathrm{kHz}$ and specimen tip base temperature of $20 \mathrm{~K}$. The occurrence of the $\mathrm{H}_{3}^{+}$ion in mass spectra during PLAP has been attributed to direct adsorption on highly protruded kink or step site atoms due to the aforementioned stability in the presence of an electric field followed by ionization and desorption [1, 46-48]. Alternatively, the formation of the $\mathrm{H}_{3}^{+}$ion has been suggested to occur by migration of the more weakly bound $\mathrm{H}_{2}$ molecule that hops onto a more strongly bound $\mathrm{H}$ atom thereby forming a $\mathrm{H}_{3}$ species that is ionized and desorbed as a $\mathrm{H}_{3}^{+}$ion $[44,49]$. However, in view of the limited expected surface diffusion at the tip apex until its temperature attains $\sim 800 \mathrm{~K}$ [33-35], the estimated short-range binding energy, and the aforesaid ionization, desorption, and dissociation model of the $\mathrm{H}_{2}^{+}$ ion suggest that the model described in Refs. [1, 46-48] may be correct.

\section{Minimizing residual $\mathrm{H}_{2}$ gas in mass spectra and applicability to other materials}

In general, this study illustrates that choosing the appropriate values of user-selected experimental parameters of laser pulse energy, laser pulse frequency, and specimen tip base temperature can appreciably minimize the quantity of residual $\mathrm{H}_{2}$ gas in the mass spectrum. The results of this study indicate that by increasing the laser pulse energy at a constant laser pulse frequency and specimen tip base temperature, the quantity of residual $\mathrm{H}_{2}$ gas in the mass spectrum can be reduced to values less than 0.1 at.\%. Moreover, the results of this study indicate that by increasing the laser pulse frequency at a constant laser pulse energy and base temperature, the quantity of residual $\mathrm{H}_{2}$ gas in the mass spectrum can be reduced. Alternatively, both pulse energy and pulse frequency can be increased at a constant base temperature to attain low values of the quantity $H_{\text {TOT }}$. Virtually all metal and alloy APT mass spectra will exhibit $\mathrm{H}$ and the results of this study on $\mathrm{Cu}$ are likely broadly applicable. The few earlier studies that evaluated single parameters in laser-pulsed mode on other metals or alloys support this observation. One previous study on Mo indicated that the $\mathrm{H}$ signal intensity decreases with increasing pulse frequency albeit at significantly slower rates [16]. Furthermore, a second previous study on a $\mathrm{Ni}-\mathrm{Cr}$-rich alloy indicated that the quantity of adsorbed $\mathrm{H}$ decreases with increasing pulse energy. The applicability to materials other than metals and alloys, such as semi-conducting materials, has not been examined in detail and needs further study in order to gain greater understanding of the behavior. For example, the APT mass spectra of cadmium telluride $(\mathrm{CdTe})$ in one recent study did not exhibit $\mathrm{H}[50,51]$. Furthermore, the study in Ref. [51] suggested the opposite trend for residual $\mathrm{H}_{2}$ gas in the mass spectra with laser pulse energy in gallium nitride $(\mathrm{GaN})$. The differences seen in these materials when compared to metals and alloys may be due to material specific properties of these compound semiconductors.

\section{Conclusions}

The presence of residual $\mathrm{H}_{2}$ gas in the mass spectra limits the ability to measure $\mathrm{H}$ concentration in metals and alloys in solid-state phenomena using APT since it is difficult to distinguish between the sources of $\mathrm{H}$ and thus needs to be minimized. The synergistic laser pulse energy, laser pulse frequency, and specimen tip base temperature influence on the field adsorption and desorption of residual $\mathrm{H}_{2}$ gas on nominally pure $\mathrm{Cu}$ was studied during UV-pulsed laser APT. This investigation resulted in the following findings:

1. Statistical analysis by ANOVA demonstrates that laser pulse energy, laser pulse frequency, and specimen tip base temperature have a significant effect on the total residual $\mathrm{H}$ concentration, $H_{\mathrm{TOT}}$. Second-order interactions of pulse energy and pulse frequency, pulse energy and base temperature, and pulse frequency and base temperature are also shown to be significant.

2. The quantity $H_{\mathrm{TOT}}$ exhibits a decreasing trend with increasing laser pulse energy between 40 and $80 \mathrm{pJ}$ at laser pulse frequencies of 100,250 , and $500 \mathrm{kHz}$ at a constant specimen tip base temperature of 20 or $50 \mathrm{~K}$.

3. The quantity $H_{\mathrm{TOT}}$ exhibits a decreasing trend with increasing pulse frequency between 100 and $500 \mathrm{kHz}$ at pulse energies of 40 and $60 \mathrm{pJ}$ and at base temperatures of 20 or $50 \mathrm{~K}$. The quantity $H_{\mathrm{TOT}}$ exhibits a generally decreasing or constant trend with increasing 
pulse frequency at a pulse energy of $80 \mathrm{pJ}$ and at base temperatures of 20, 50, and $80 \mathrm{~K}$.

4. Laser pulse energy has the greatest effect on the quantity $H_{\mathrm{TOT}}$, which is less than 0.1 at.\% at a pulse energy of $80 \mathrm{pJ}$, and pulse frequencies of 100,250 , or $500 \mathrm{kHz}$, and base temperatures of 20,50 , or $80 \mathrm{~K}$. The pulse frequency and base temperature have only a smaller effect on the quantity $H_{\mathrm{TOT}}$ at a $80 \mathrm{pJ}$ pulse energy when compared to a 40 or $60 \mathrm{pJ}$ pulse energy.

5. The results of this study indicate that the quantity of residual $\mathrm{H}_{2}$ gas can be minimized in mass spectra by increasing the laser pulse energy at a constant laser pulse frequency or specimen tip base temperature. The quantity of residual $\mathrm{H}_{2}$ gas can also be minimized in mass spectra by increasing the laser pulse frequency at a constant laser pulse energy and base temperature. Alternatively, both pulse energy and pulse frequency can be increased at a constant base temperature.

\section{Abbreviations}

APT: atom probe tomography; UV: ultraviolet; 1D: one-dimensional; APFIM: atom probe field ion microscope; PLAP: pulsed laser atom probe; LEAP: localelectrode atom probe; UHV: ultra-high vacuum; IVAS: integrated visualization and analysis software; ANOVA: analysis of variance; 2D: two-dimensional; f.c.c.: face-centered cubic; b.c.c.: body-centered cubic; h.c.p.: hexagonal close-packed.

\section{Acknowledgements}

The author would like to thank Dr. Frederick Meisenkothen and Mr. Eric B. Steel of the National Institute of Standards and Technology (NIST) for access to the LEAP $^{\circledR} 4000 X$ Si instrument. Dr. Frederick Meisenkothen is also thanked for his helpful discussions and reviewing the manuscript. Mr. Samuel C. Schwarm of the University of Maryland (UMD) is thanked for reviewing the manuscript. Dr. Babak Nikhoobhakt of NIST is thanked for his assistance with the FEI Nova 600 dual-beam SEM/FIB instrument and his helpful discussions regarding specimen preparation.

\section{Competing interests}

The author declare that he has no competing interests.

\section{Availability of data and materials}

The datasets supporting the conclusions of this article are available at https:// figshare.com.

Received: 28 September 2016 Accepted: 10 February 2017

Published online: 22 February 2017

\section{References}

1. Tsong, T.T., Kinkus, T.J.: Energy distributions of pulsed-laser field-desorbed gaseous ions and field-evaporated metal ions: a direct time-of-flight measurement. Phys. Rev. B. 29, 529-542 (1984)

2. Gault, B., Moody, M.P., Cairney, J.M., Ringer, S.P.: Atom Probe Microscopy. Springer, New York (2012)

3. Larson, D.J., Prosa, T.J., Ulfig, R.M., Geiser, B.P., Kelly, T.F.: Local Electrode Atom Probe Tomography_A User's Guide. Springer, New York (2013)

4. Gemma, R., Al-Kassab, T., Kirchheim, R., Pundt, A.: Visualization of deuterium dead layer by atom probe tomography. Scripta Mater. 67, 903-906 (2012)

5. Takahashi, J., Kawakami, K., Kobayashi, Y., Tarui, T.: The first direct observation of hydrogen trapping sites in TiC precipitation-hardening steel through atom probe tomography. Scripta Mater. 63, 261-264 (2010)
6. Takahashi, J., Kawakami, K., Tarui, T.: Direct observation of hydrogentrapping sites in vanadium carbide precipitation steel by atom probe tomography. Scripta Mater. 67, 213-216 (2012)

7. Haley, D., Merzlikin, S.V., Choi, P., Raabe, D.: Atom probe tomography observation of hydrogen in high-Mn steel and silver charged via an electrolytic route. Int. J. Hydrogen Energy 39, 12221-12229 (2014)

8. Sundell, G., Thuvander, M., Yatim, A.K., Nordin, H., Andrén, H.-O.: Direct observation of hydrogen and deuterium in oxide grain boundaries in corroded zirconium alloys. Corros. Sci. 90, 1-4 (2015)

9. Takamizawa, H., Hoshi, K., Shimizu, Y., Yano, F., Inoue, K., Nagata, S., Shikama, T., Nagai, Y.: Three-dimensional characterization of deuterium implanted in silicon using atom probe tomography. Appl. Phys. Express 6 $66602(2013)$

10. Andrén, H.-O., Rolander, U.: Field dependence of hydrogen adsorption. Surf. Sci. 266, 76-80 (1992)

11. Macrander, A.T., Seidman, D.N.: Hydrogen adsorption on (110) tungsten at $30 \mathrm{k}$ : an atom-probe field-ion microscope study. Surf. Sci. 147, 451-465 (1984)

12. Hellsing, M., Hellsing, B.: Field adsorption and desorption of hydrogen on W(110) -an atom-probe study. Surf. Sci. 176, 249-260 (1986)

13. Nishikawa, O., Yoshimura, T., Shibata, M.: Atom-probe study of hydrogen physisorption on Al and Cu. Surf. Sci. 124, 440-450 (1983)

14. Wada, M., Uemori, R., Nishikawa, O.: Effect of hydrogen on the evaporation field of metals. Surf. Sci. 134, 17-29 (1983)

15. Nishikawa, O.: Reduced evaporation field by the field induced dipoles of physisorbed $\mathrm{He}, \mathrm{Ne}$ and $\mathrm{H}_{2}$. Surf. Sci. 131, 239-244 (1983)

16. Kellogg, G.L.: Pulsed laser stimulated field desorption of hydrogen from molybdenum. J. Chem. Phys. 74, 1479-1487 (1981)

17. Drachsel, W., Block, J.H.: Isotopic effects in field desorption of hydrogen from tungsten. Surf. Sci. 246, 141-149 (1991)

18. Sundell, G., Thuvander, M., Andrén, H.-O.: Hydrogen analysis in APT: methods to control adsorption and dissociation of $\mathrm{H}_{2}$. Ultramicroscopy 132 , 285-289 (2013)

19. Kelly, T.F., Larson, D.J.: Atom probe tomography 2012. Annu. Rev. Mater. Res. 42, 1-31 (2012)

20. Devaraj, A., Perea, D.E., Liu, J., Gordon, L.M., Prosa, T.J., Parikh, P., Diercks, D.R., Meher, S., Kolli, R.P., Meng, Y.S., Thevuthasan, S.: Three-dimensional nanoscale characterisation of materials by atom probe tomography. Int Mater. Rev. (2017). doi:10.1080/09506608.2016.1270728

21. Tsong, T.T., Müller, E.W.: Field adsorption of inert-gas atoms on field ion emitter surfaces. Phys. Rev. Lett. 25, 911-913 (1970)

22. Tsong, T.T., Müller, E.W.: Field adsorption of inert-gas atoms. J. Chem. Phys. 55, 2884-2889 (1971)

23. Thompson, K., Lawrence, D., Larson, D.J., Olson, J.D., Kelly, T.F., Gorman, B.: In situ site-specific specimen preparation for atom probe tomography. Ultramicroscopy 107, 131-139 (2007)

24. Miller, M.K., Russell, K.F., Thompson, K., Alvis, R., Larson, D.J.: Review of atom probe fib-based specimen preparation methods. Microsc. Microanal. 13, 428-436 (2007)

25. Kolli, R.P., Meisenkothen, F.: A focused ion beam specimen preparation method to minimize gallium ion concentration in copper atom-probe tomography specimen tips. Microsc. Microanal. 20, 350-351 (2014)

26. Thompson, K., Gorman, B., Larson, D., van Leer, B., Hong, L.: Minimization of Ga induced FIB damage using low energy clean-up. Microsc. Microanal. 12, 1736-1737 (2006)

27. Saxey, D.W.: Correlated ion analysis and the interpretation of atom probe mass spectra. Ultramicroscopy 111, 473-479 (2011)

28. Meisenkothen, F., Steel, E.B., Prosa, T.J., Henry, K.T., Prakash Kolli, R.: Effects of detector dead-time on quantitative analyses involving boron and multi-hit detection events in atom probe tomography. Ultramicroscopy 159, 101-111 (2015)

29. Kolli, R.P., Meisenkothen, F.: Optimization of experimental parameters and specimen geometry for pulsed laser atom-probe tomography of copper. Microsc. Microanal. 20, 768-769 (2014)

30. Kolli, R.P., Meisenkothen, F.: The influence of experimental parameters and specimen geometry on the mass spectra of copper during pulsed-laser atom-probe tomography. Microsc. Microanal. 20, 1715-1726 (2014)

31. Vurpillot, F., Houard, J., Vella, A., Deconihout, B.: Thermal response of a field emitter subjected to ultra-fast laser illumination. J. Phys. D Appl. Phys. 42 , 125502 (2009)

32. Kellogg, G.L., Tsong, T.T.: Pulsed-laser atom-probe field-ion microscopy. J. Appl. Phys. 51, 1184 (1980) 
33. Kellogg, G.L.: Determining the field emitter temperature during laser irradiation in the pulsed laser atom probe. J. Appl. Phys. 52, 5320 (1981)

34. Cerezo, A., Smith, G.D.W., Clifton, P.H.: Measurement of temperature rises in the femtosecond laser pulsed three-dimensional atom probe. Appl. Phys. Lett. 88, 154103 (2006)

35. Cerezo, A., Clifton, P.H., Gomberg, A., Smith, G.D.W.: Aspects of the performance of a femtosecond laser-pulsed 3-dimensional atom probe. Ultramicroscopy 107, 720-725 (2007)

36. Vurpillot, F., Gault, B., Vella, A., Bouet, M., Deconihout, B.: Estimation of the cooling times for a metallic tip under laser illumination. Appl. Phys. Lett. 88, 94105 (2006)

37. Forbes, R.G.: On charged-surface models and the origin of field adsorption. Surf. Sci. 223, 326-352 (1989)

38. Wang, R.L.C., Kreuzer, H.J., Forbes, R.G.: Field adsorption of helium and neon on metals: an integrated theory. Surf. Sci. 350, 183-205 (1996)

39. Forbes, R.G.: Further comments on field adsorption. Surf. Sci. 246 386-389 (1991)

40. Knickelbein, M.B.: Electric dipole polarizabilities of copper clusters. J. Chem. Phys. 120, 10450-10454 (2004)

41. Slater, J.C.: Atomic radii in crystals. J. Chem. Phys. 41, 3199-3204 (1964)

42. Madhavan, P.V., Whitten, J.L.: Hydrogen adsorption on copper: embedding theory based on orbital localization. Surf. Sci. 112, 38-51 (1981)

43. Forni, A., Wiesenekker, G., Baerends, E.J., Tantardini, G.F.: The chemisorption of hydrogen on Cu(111): a dynamical study. Int. J. Quantum Chem. 52 1067-1080 (1994)
44. Ye, X., Kreuzer, H.J., Salahub, D.R.: Theory of field adsorption of hydrogen. Appl. Surf. Sci. 67, 1-8 (1993)

45. Reckzügel, M.C., Drachsel, W., Block, J.H.: Field desorption of $\mathrm{H}_{3}$ and field dissociation of $\mathrm{H}^{3+}$. Appl. Surf. Sci. 76-77, 108-114 (1994)

46. Tsong, T.T., Kinkus, T.J., Ai, C.F.: Field induced and surface catalyzed formation of novel ions : a pulsed-laser time-of-flight atom-probe study. J. Chem. Phys. 78, 4763-4775 (1983)

47. Ai, C.F., Tsong, T.T.: A study of the temperature dependence of a surface catalyzed and field enhanced formation of $\mathrm{H}_{3}$ and $\mathrm{NH}_{3}$ on metal surfaces. J. Chem. Phys. 81, 2845-2854 (1984)

48. $\mathrm{Ai}$, C.F., Tsong, T.T.: Field promoted and surface catalyzed formation of $\mathrm{H}_{3}$ and $\mathrm{NH}_{3}$ on transition metal surfaces: a pulsed-laser imaging atom-probe study. Surf. Sci. 138, 339-360 (1984)

49. Ernst, N., Block, J.H.: Temperature programmed field desorption of protonated hydrogen from rhodium and tungsten. Surf. Sci. 126, 397-404 (1983)

50. Choi, P.-P., Cojocaru-Mirédin, O., Abou-Ras, D., Caballero, R., Raabe, D., Smentkowski, V.S., Park, C.G., Gu, G.H., Mazumder, B., Wong, M.H., Hu, Y.-L., Melo, T.P., Speck, J.S.: Atom probe tomography of compound semiconductors for photovoltaic and light-emitting device applications. Microsc. Today 20, 18-24 (2012)

51. Diercks, D.R., Gorman, B.P.: Nanoscale measurement of laser-induced temperature rise and field evaporation effects in CdTe and GaN. J. Phys. Chem. C 119, 20623-20631 (2015)

\section{Submit your manuscript to a SpringerOpen ${ }^{\odot}$ journal and benefit from:}

- Convenient online submission

- Rigorous peer review

- Immediate publication on acceptance

- Open access: articles freely available online

- High visibility within the field

- Retaining the copyright to your article 